\title{
Apprehension of pictorial events: The case of a moving observer in a static environment
}

\author{
JOHN B. PITTENGER \\ University of Arkansas, Little Rock, Arkansas 72204 \\ and \\ JAMES J. JENKINS \\ University of Minnesota, Minneapolis, Minnesota 55455
}

\begin{abstract}
Slides of views along a walk through a campus were shown to observers. One week later, some of these slides, plus new views from the walk and similar control slides, were presented for confidence-weighted recognition judgments. Appropriate new scenes but not controls were falsely recognized with high frequency and confidence. Strength of these recognitions relative to those of slides actually shown earlier depended on the observer's familiarity with the campus and the order of slides in the original presentation. Results are interpreted in terms of the apprehension of events on the basis of information available in the relations among discrete scenes.
\end{abstract}

Until recently, research in perception and memory has focused on discrete, static material: line drawings, single words, pure tones, and so on. While a number of stimulus items are generally used, they are often unrelated in the sense that they do not form a coherent whole. To account for memory of more complex material such as visual events, connected prose, and so on, constructive processes operating on the static units are often postulated. The present research proceeds from a different basic assumption, namely, that events rather than static patterns should be the primary focus of cognitive psychology. Events involve both objects and changes (i.e., transformations) defined over the objects. From this point of view, changes that relate discrete items are fundamental rather than derived concepts. Objects may be defined, in part, by aspects left invariant by certain transformations: In Piagetian theory, for example, a key aspect of objects, the quantity of material, is definable only in terms of the physical operations, transformations, that leave the quantity invariant. Similarly, the perceived identity of a person's face does not lie in any single momentary view of the face, but, rather, in characteristics left invariant over the changes in emotional expression,

This research was supported by grants to the Center for Research in Human Learning, University of Minnesota, from the National Science Foundation (GB17590), the National Institu te for Child Health and Human Development (HD 01136), the Graduate School, University of Minnesota, and by a travel grant from the Donaghey Foundation. We thank Jerry Wald for criticism and comments. Requests for reprints may be sent to John Pittenger, Department of Psychology, University of Arkansas, Little Rock, Arkansas 72204. effects of aging, and so on. This point of view is more fully developed in Shaw and Pittenger (1978).

This viewpoint has strong consequences for the stimulus materials, design, and interpretations of empirical research. In perception, Michotte's (1963) work on perceived causality and Johansson's (1973) work on biological motion demonstrate the existence of perceived events in dynamic stimuli not predictable from static views alone, but only from the dynamic, transformational aspects.

The application of these views to memory research can be illustrated by comparing several experiments. Several researchers, Shepard (1967), and Standing and his colleagues (Standing, 1973; Standing, Conezio, \& Haber, 1970), presented thousands of unrelated pictures and found extremely accurate recognition memory. Goldstein and Chance (1970) applied similar procedures to sets of items related by membership in a class: sets of snowflakes, faces of different individuals, and various inkblot patterns. With such categorically related stimuli, recognition accuracy was dramatically lower. From a point of view emphasizing events, a parallel situation should arise with stimuli related by their participation in an event rather than by category membership. Suppose observers are first presented with a number of pictures of an event in progress and then shown a set of recognition slides including some of those photographs shown previously, plus other pictures from the same event and visually similar pictures that are not scenes from the event. If new scenes from the event are correctly rejected, one may conclude that event structure has little relevance to recognition memory. On the other hand, if the new scenes are falsely recognized and the 
scenes not from the event are correctly rejected, then the event has influenced recognition performance. The point of view taken in this paper interprets the false recognitions not merely as errors but rather as evidence that the meaningful relations specified in the stimuli to be remembered were apprehended and form part of the memory.

The event used in the present studies was a walk through a college campus. The stimuli that specified the event were slides of what an observer would see looking straight ahead during the walk. Control stimuli included slides of views of the campus from station points off the paths of motion and from points on the path but in directions perpendicular to the direction of motion.

This event, in which only the observer's station point changes over time, is only one of several basic types of events (cf. Gibson, 1966). The observer could remain stationary and view motions of objects, or both the observer and objects in the scene might move. These different types of events involve different changes over time, and, therefore, provide different types of information to the observer. Research on other classes of events, as well as the studies reported here, are briefly described in Jenkins, Wald, and Pittenger (1978).

While the event in question, and the particular views photographed, determine the information available, the observer's access to that information can be manipulated in two ways: order of presentation of the memorization set and observer's knowledge of the campus. Presenting a subset of the event slides in the order they would be seen by an actual walker might be expected to make the event particularly clear. This could maximize false recognitions of new event slides by strengthening the event relative to specific memory for individual slides or could reduce them by providing a framework that would make the novelty of new views particularly obvious. Random ordering of the memorization items might destroy the event by its violation of the temporalspatial pattern. The constraints among the slides are, however, fairly strong, and the event might be reconstructed. For example, a series of photos of an orange being peeled would be hard to mistake no matter what the presentation order.

The observer's knowledge of the campus is another type of information relevant to the event. Its effect was separated from the information in the slides themselves by comparing the responses of subjects unfamiliar with the campus with those of resident students.

The effect of the "density" of information in the memorization set was tested by leaving a "gap" in the sequence. For one section of about 30 yards, no slides were shown. For observers, this omission might specify that the event did not include this section of the path. Alternatively, it might have no effect on the perceived event. There was a good deal of distance between station points for other slides, and the "gap" might be taken as simply an unusually large distance between samples of the event.

\section{EXPERIMENT 1}

\section{Method}

Stimuli. A large set of color slides was taken of the campus of the University of Rhode Island. The event consisted of views taken about every 20 steps along a walk from the student union to the psychology building and back. In particular, the slides showed what the walker would see looking straight ahead, along the path of motion. The path was fairly complex: out of the union, a 90-deg turn toward the library, a short dog-leg on the path to the library, a 45-deg turn onto a diagonal past the library, a 45-deg turn off the diagonal, across a street, and a 45-deg turn up the entrance ramp to the psychology building. The return to the union used the same path and showed the straight-ahead view seen by the walker. Control slides were of three types: side views taken while on the path but $90 \mathrm{deg}$ from the path of motion, views of buildings visible from the path but taken from other station points off the path, and views of other areas of the campus. The slides were taken on a clear sunny Sunday when the campus was virtually deserted. Thus, such distinguishing characteristics as appearance and disappearance of people, autos, and so on, did not occur in the set.

The memorization set consisted of a subset of the event slides, that is, straight-ahead front views along the path of motion. These were, with one exception, distributed fairly uniformly along the path to and from the psychology building. The exception was that no slides were shown of the diagonal path around the library on the return to the union. This part of the event will be referred to as the gap. Of the 13 slides in this memorization set, 9 showed views walking toward the psychology building and 4 showed the return to the union.

The recognition set included 23 slides of four types: 7 originals, slides shown in the memorization phase; 7 belonging, slides from the event but not shown in memorization; 7 controls, slides not part of the event (including side views along the path); 2 gap, slides from the event but in the segment not represented in the memorization set.

Subjects. Thirty University of Rhode Island students enrolled in introductory psychology participated for course credit.

Procedure. All subgroups followed the same basic procedure. In the memorization phase, the set of 13 slides was shown twice at a rate of $7.5 \mathrm{sec} / \mathrm{slide}$. Subjects were instructed to study the slides so that they could recognize them in the second session. They were not told about the event involved in the set but were simply told that views of a college campus would be shown. Subjects returned in 1 week for the recognition task. Subjects were asked to decide for each slide whether it was "old," meaning that it had been shown in the first phase, or "new," meaning that it was being shown for the first time. They were also asked to rate their confidence in each judgment. Subjects marked their responses on an 11-point scale. One end of the scale was marked "old" and included five levels of confidence ranging from "certain" to "weakly confident." The other end was marked "new," with the same five levels of confidence. The center of the scale was marked "guess" and was to be used when a subject could not choose between "old" and "new" with even weak confidence. The slides were shown for about $10 \mathrm{sec}$ each.

Fifteen subjects viewed the memorization set in the event sequence, that is, in the order the slides would be seen when taking the walk, and 15 saw them in randomized orders. Each group was tested in three subgroups of five subjects. The set of slides was presented twice to each subgroup. Each subgroup in the random group viewed the slides in different random sequences. All subgroups in the event condition viewed the slides in the event order. The order was the same for the two presentations within each subgroup. The recognition set was presented only once. Presentations to each subgroup within a condition were in a different random order, the same three orders being used across conditions. Recognition instructions stressed the 
fact that there would be great similarities among the slides and it was important to be careful to respond "old" only to those slides actually seen in the first phase.

\section{Results and Discussion}

The mean recognition ratings over subjects and slides within type and the percent "old" judgments for each type of test slide are presented in Table 1. "Old" judgments were scored from 11 to 7 , with 11 as maximum and 7 as minimum confidence; "new" judgments were scored from 5 to 1 , with 5 as the minimum and 1 as maximum confidence. "Guess" judgments were scored as 6.

The key comparisons are among the original, belonging, and control slides. Both the mean ratings and percent "old" judgments indicate that original and belonging slides were confidently recognized as being shown in the first phase. Control slides were rarely mistaken for old scenes. Additionally, subjects were virtually unable to distinguish between original and belonging slides. Using the mean rating of slides of a single type as the index of recognition of that type, both original and belonging slides were more highly rated than controls on sign tests for matched pairs. For each of the 30 subjects, the means for both original and belonging slides were larger than the mean for controls $(\mathrm{z}=7.9, \mathrm{p}<.001$, two-tailed matched-pairs sign test). A sign test of the hypothesis that original slides were more highly rated than belonging slides did not reach significance; for 17 subjects the mean for originals was larger, for 12 it was smaller (one tie) $(z=.93)$. Thus the main prediction, that subjects would falsely recognize new slides from the event but correctly reject similar controls, was strikingly confirmed. The event, the change in station point that ties the event slides together, appears to be a major determiner of recognition performance. Actual viewing of the slides in the memorization phase contributed little to the confidence in the "old" judgments.

This basic pattern of results held for both random and event sequence presentation orders. Breaking the temporal order of the event did not destroy the effect of the event on recognition performance. This result is not surprising in view of the fact that little else could be "made of" the set as a group. When questioned after the experiment, subjects in the random group usually stated that the memorization set involved the route between the two buildings. There did, however, appear to be an effect of presentation order. The ratings of the

Table 1

Mean Ratings and Percent “Old" (0) Judgments in Experiment 1

\begin{tabular}{lccccccccc}
\hline & \multicolumn{8}{c}{ Stimulus Type } \\
\cline { 2 - 9 } $\begin{array}{c}\text { Presentation } \\
\text { Condition }\end{array}$ & Mean & O & Mean & O & Mean & O & Mean & O \\
\hline Random & 8.38 & 76 & 7.50 & 68 & 2.31 & 8 & 5.00 & 20 \\
Event Sequence & 8.65 & 82 & 8.57 & 83 & 2.70 & 11 & 5.10 & 27 \\
\hline
\end{tabular}

Note-There were seven slides of each type, except for the gap, which included two slides. belonging slides for subjects in the two presentations were compared in a Mann-Whitney $U$ test on the hypothesis that the belonging slides were more confidently recognized after the event order presentation than after random presentation. The resulting $U=185$ gives $\mathrm{z}=2.59(\mathrm{p}<.01)$. This suggests that presenting the slides in the event sequence strengthened the cohesion of the event, making old and new elements of the event harder to distinguish, rather than providing a framework that would aid memory for specific scenes.

The slides in the "gap" were poorly recognized: The mean ratings are in the "new" range and only about a quarter of the judgments were of "old." On a sign test of the hypothesis that belonging slides were rated higher than gap slides, the mean belonging rating was higher for 27 subjects and lower for $3(\mathrm{z}=4.38$, $\mathrm{p}<.001)$. On the other hand, gap slides are rated higher than the controls; the mean of gap slides was higher than the mean for controls for 25 subjects and lower for $5(\mathrm{z}=3.65, \mathrm{p}<.001)$.

In Experiment 1 false recognitions could have been influenced by both the visual information in the stimulus set and the subjects' own knowledge of the physical layout of the campus. Experiment 2 attempted to separate these variables by presenting the slides in the random and ordered conditions to a similar population of students at a distant university.

\section{EXPERIMENT 2}

\section{Method}

The method was identical to that in Experiment 1 except that the 30 subjects, University of Arkansas at Little Rock undergraduates participating for course credit, had never visited or seen pictures of the Rhode Island campus. They therefore had no knowledge of the buildings, walkways, and so on, of the campus shown in the slides.

\section{Results and Discussion}

The mean ratings and percent "old" judgments for Experiment 2 are presented in Table 2.

In contrast to Experiment 1, the original slides were rated significantly higher than belonging slides; for 28 subjects the mean on originals was greater than the mean on belonging slides, while for 2 they were smaller (two-tailed matched-pairs sign test, $\mathrm{z}=4.75, \mathrm{p}<.001$ ). Thus, subjects without prior knowledge of the campus were better able to discriminate between previously shown and new event slides than were knowledgeable subjects. Nonetheless, the belonging slides were more

Table 2

Mean Ratings and Percent “Old" (0) Judgments in Experiment 2

\begin{tabular}{lccccccccc}
\hline & \multicolumn{8}{c}{ Stimulus Type } \\
\cline { 2 - 9 } \multicolumn{1}{c}{$\begin{array}{c}\text { Presentation } \\
\text { Condition }\end{array}$} & Mean & O & Mean & O & Mean & O & Mean & O \\
\hline Random & 9.44 & 89 & 7.76 & 66 & 2.26 & 9 & 5.20 & 37 \\
Event Sequence & 9.27 & 85 & 6.55 & 54 & 2.01 & 4 & 3.90 & 23 \\
\hline
\end{tabular}

Note-There were seven slides of each type, except for the gap, which included two slides. 
often than not rated as "old" and were significantly more highly rated than were controls. For each of the 30 subjects, the mean for belonging slides was greater than the mean for controls (as in Experiment 1, z = 7.9, $\mathrm{p}<.001$ ). Identical results were found for a sign test on the difference between originals and controls. Thus, while knowledge of the campus is a factor in how strongly the event influences recognition performance, the information in the memorization set itself is able to produce false recognition of the novel event slides.

In Experiment 1, showing the memorization set in event sequence order raised the ratings of belonging slides relative to random order. For subjects not familiar with the campus, the effect tends to be the reverse. A Mann-Whitney U test fails, however, to reach significance $(U=151, z=1.6, p>.05)$. For knowledgeable subjects, it was concluded that event ordering of the input tended to "fuse" the event and make novel event stimuli less discriminable from previously presented event stimuli. With naive subjects, this does not occur and may even make the new event stimuli more obvious. The present data are not, however, strong enough to be conclusive.

Finally, the ratings of the gap slides are again between those for the belonging and control slides. For 28 subjects the belonging mean was greater than the gap mean $(z=4.02, p<.01$, for the sign test $)$, and for 24 subjects the gap mean was greater than the control mean $(\mathrm{z}=3.29, \mathrm{p}<.01)$.

\section{GENERAL DISCUSSION}

The results of these experiments show that natural events protrayed in a series of slides can be apprehended by observers. When apprehended, the events strongly influence the recognition responses. While subjects are certain that the visually similar control slides had not been shown earlier, they generally believe the new belonging slides are repetitions from the past. They are almost as certain of these slides as they are of the ones actually shown.

Violation of the natural order of scenes and the subjects' knowledge of the event had significant influences on the recognition ratings. The size of the influence was only moderate. While there were changes in the relations between original and belonging slides, the higher rate of recognition of belonging slides over controls persisted. For subjects familiar with the campus, that is, the framework in which the event occurred, it was harder to discriminate between previously shown and new event slides than for naive subjects. Order and knowledge also showed an interactive effect. For those familiar with the campus, showing the memorization set in the order of the event increased the false recognition rate of the new event slides. For naive subjects the difference, though nonsignificant, was in the reverse direction. A "gap" in the input set, providing essentially ambiguous information about the event in that section, appears to produce ambiguous recognition judgments. In summary, stimuli related by their participation in an event of the type of an observer moving through a static environment influence recognition performance very powerfully. The nature of those influences, while not yet predictable a priori, are readily interpretable.

The perspective of event specification in stimuli also suggests some extensions of other work in memory. In recent years, de Groot's (1965) work on the role of chess knowledge on memory for position of pieces in random and in game boards has been extended (cf. Charness, 1976; Chase \& Simon, 1973). These studies have focused on one board for each of a number of games. The board at any single moment in time partially specifies an event, the ongoing game. It is relatively easy to propose a variety of studies using a number of different boards for a single game. Such studies may aid the analysis of chess skill being done with single boards by increasing the amount of information relevant to play-the dynamic aspect. Mandler and her colleagues (Mandler \& Johnson, 1976; Mandler \& Parker, 1976) have studied types of information available in individual pictures by using a variety of transformations of previously shown pictures as foils in a recognition task. One important type of information not available in the pictures used is information for a specific event. The pictures do show a loosely specified event, such as a teacher lecturing or children at a picnic. However, the people and objects are not arranged spatially so as to be interacting in particular, specific ways. The inclusion of such interactions is likely to affect strongly subjects' responses to changes in spatial relations, replacement of objects, and so on, in the foils.

It is important for research and theory in memory to account for the complexities of real-world situations. Conceiving of some situations as temporally and spatially extended events has been helpful in the area of perception and should be considered for work in memory. The present results, and others reported in Jenkins et al. (1978), show that event relations do affect behavior in memory tasks and illustrate one convenient method of analyzing the influence of variables relevant to the nature of information for the event.

\section{REFERENCES}

Charness, N. Memory for chess positions: Resistance to interference. Journal of Experimental Psychology: Human Learning and Memory, 1976, 2, 641-653.

Chase, W. G., \& Simon, H. A. Perception in chess. Cognitive Psychology, 1973, 4, 55-81.

DE GROOT, A. Thought and choice in chess. The Hague: Mouton, 1965.

GiBson, J. J. The senses considered as perceptual systems. Boston: Houghton Mifflin, 1966.

Goldstein, A. G., \& Chance, J. E. Visual recognition memory for complex configurations. Perception \& Psychophysics, 1970, 9, 237-241.

Jenkins, J. J., Wald, J., \& Pittenger, J. B. Apprehending pictorial events: An instance of psychological cohesion. In C. W. Savage (Ed.), Minnesota studies in the philosophy of science (Vol. 9). Minneapolis: University of Minnesota Press, 1978.

JoнAnsson, G. Visual perception of biological motion and a model for its analysis. Perception \& Psychophysics, 1973, 14, 201-211.

MANDler, J. M., \& Jornson, N. S. Some of the thousand words a picture is worth. Journal of Experimental Psychology: Human Learning and Memory, 1976, 2, 529-540.

Mandler, J. M., \& Parker, R. E. Memory for descriptive and spatial information in complex pictures. Journal of Experimental Psychology: Human Learning and Memory, 1976, 2 , 38-48.

Michorte, A. The perception of causality. London: Methuen, 1963.

Shaw, R. E., \& Prtrenger, J. B. On perceived change. In H. Pick \& E. Saltzman (Eds.), Modes of perceiving and processing information. Hillsdale, N.J: Erlbaum, 1978, 187-204.

SHEPARD, R. N. Recognition memory for words, sentences and pictures. Journal of Verbal Learning and Verbal Behavior, 1967, 6, 156-163.

Standing, L. Learning 10,000 pictures. Quarterly Journal of Experimental Psychology, 1973, 25, 207-222.

Standing, L., Conezio, J., \& Haber, R. N. Perception and memory for pictures: Single-trial learning of 2500 visual stimuli. Psychonomic Science, 1970, 19, 73-74.

(Received for publication January 3, 1979.) 